\title{
Efecto de la Actividad Física y Desempeño del Aprendizaje Hipocampal
}

\section{Effect of Physical Activity and Hippocampal Learning Performance}

Juan David Almarales-Sanabria ${ }^{1}$

Recibido: 17/diciembre/2020

Aceptado: 6/abril/2021

Publicado: $16 /$ abril/2021

\&aís

${ }^{1}$ Colombia

\section{IIIII Institución}

${ }^{1}$ Instituto de la juventud, el deporte y la recreación de Bucaramanga

\section{\Correo Eletrónico \\ 1jalmarales@unab.edu.co}

\section{iD ORCID}

'https://orcid.org/0000-0002-8250-5816

\section{Citar así: C APA / IEEE}

Almarales-Sanabria, J. (2021). Efecto de la actividad física $y$ desempeño del aprendizaje hipocampal. Revista Tecnológica-Educativa Docentes 2.0, 11(1),

https://doi.org/10.37843/rted.v11i1.191

J. Almarales-Sanabria, "Efecto de la actividad física y desempeño del aprendizaje hipocampal", RTED, vol. 11, n. ${ }^{\circ} 1$, pp. 62-67, abr. 2021.

\section{Resumen}

El estilo de vida del hombre se ha caracterizado desde sus comienzos por las diferentes manifestaciones de actividad física en su quehacer diario. Un referente para contextualizar su importancia en el ámbito universitario, para contrastar las variables de conductas nocivas como hábitos de estudios inadecuados, baja capacidad de comprensión en ambientes académicos como factores incidentes en el rendimiento académico por parte de los estudiantes. El objetivo de dicho trabajo describió el efecto de la actividad física como el comportamiento hipocampal asociado al rendimiento académico en estudiantes universitarios de facultades de cultura física, deporte, recreación, seccional Bucaramanga. Dicho estudio se diseñó con énfasis cuasi experimental, se planteó como un protocolo de intervención de un mes a través de plataformas digitales interactivas $\mathrm{n}$ conjunto con un software especializado (MATLAB), mediante el cual un sistema de cómputo numérico dio pie a un entorno de desarrollo integrado con un lenguaje de programación propio, adaptable a otras plataformas como Windows. En tal ocasión, 57 participantes sanos desarrollaron a través de un serial de códigos, diferentes patrones evaluables, la medición de las capacidades del aprendizaje dependiente del hipocampo, en una tarea de búsqueda visual "T", en correlación con el efecto de variables de actividad física regulada con su alta demanda de oxígeno a nivel fisiológico o cerebral.

Palabras clave: Actividad física, neurociencia, educación.

\section{Abstract}

The lifestyle of man has been characterized since inception by the different manifestations of physical activity in their daily work. A reference to contextualize the importance of it in the university environment is to contrast the variables of harmful behaviors such as inappropriate study habits and low capacity for understanding in academic settings as factors that affect academic performance by students. The objective of this work described the effect of physical activity as hippocampal behavior associated with a theoretical account in university students from faculties of material culture, sports, recreation, Bucaramanga section. This study was designed with a quasi-experimental emphasis as a one-month intervention protocol through interactive digital platforms Microsoft teams, in conjunction with specialized software (MATLAB). A numerical computing system gave rise to an Integrated development environment with its programming language, adaptable to other platforms such as Windows. On this occasion, 57 healthy participants developed, through a series of codes, different evaluable patterns, the measurement of hippocampal-dependent learning abilities in a visual search task "T," in correlation with the effect of activity variables. Physics is regulated with its high oxygen demand at the physiological or cerebral level.

Keywords: Technology, education, TAC, teaching, technoemotional. 


\section{Introducción}

El estilo de vida del hombre se ha caracterizado desde sus comienzos por las diferentes manifestaciones de actividad física en su quehacer diario. Hoy en día, poblaciones universitarias se han visto afectadas por disminución en los niveles de esta, dada su conducta no saludable, impidiendo el desarrollo de los diferentes componentes condiciónales físicos (Polo-Zegarra, 2020). Ante esta situación, es más evidente la carencia de estudios de este tipo a nivel local (Bucaramanga), por ende, surgen investigaciones enfocadas en conocer de forma precisa, cuáles son los efectos generados por la actividad física o el desempeño en el aprendizaje dependiente del hipocampo en estudiantes universitarios (Rojas, Salas \& Jimenez, 2006).

Históricamente se puede llegar apreciar una considerable presencia de condición física en todas las actividades (entendidas estas a nivel físico o motriz) del ser humano propiamente. (Duque-Fernández, Ornelas-Contreras \& Benavides-Pando, 2020). A lo largo del tiempo el estilo de vida (actividades de la vida diaria) ha evidenciado una fuerte relación entre condición física y salud, dado el cómo se aglutinan en un conjunto muy amplio de factores biológicos, personales o socioculturales. Debido al énfasis para entender los mismos, se establecen aspectos fundamentales al determinar capacidades funcionales de las personas. Según Anilema (2020) cuanta más actividad física se realice, mayores serán los niveles de condición física de una persona. Sin duda alguna, cualquier clase de disminución se contrastará en los tipos de actividad física establecida en principal relación a insuficiencia en las capacidades mostradas por los condicionantes físicos (Astudillo-García \& Rojas-Russell, 2006).

Una de las cuestiones más alarmantes en poblaciones universitarias, están directamente asociadas $o$ propensas a cambiar su desenvolvimiento conductual, llegando a un estilo de vida no saludable como determinantes de organización o sociedad (Jiménez, 2007). Los rangos de prevalencias encontrados en cada uno de los factores de riesgo comportamentales son significativos en población universitaria, comparados a la población en general
(Guadalupe, Amaya \& Condo, 2015)

A su vez, se destacan necesidades de fortalecer los esfuerzos por parte de los gobiernos en las comunidades universitarias, con fines de reducir las prevalencias de estas conductas nocivas, implementando medidas en políticas públicas, estrategias no solo de intervención, sino de promoción al desarrollo de estilos de vida saludables (Caballero, Delgado, Arenas, Díaz \& López,2020).Lo anterior conduce al sistema de salud pública a la implementación y rápido desenvolvimiento en términos de prevención, acción para contrarrestar los efectos de las conductas nocivas generadas en los estilos de vida de las personas, pues de esta forma se lograría controlar el posterior desarrollo de enfermedades no transmisibles. Es importante participar de los gobiernos o comunidades universitarias para crear estrategias de intervención novedosas incentivando, el mantenimiento de estilos de vida saludable o en su defecto adquirir uno.

Se destaca en este punto, diferencias entre las dos definiciones mencionadas, dado su orientación hacia el significado de condición física, el cual tradicionalmente, ha sido deportivo, encaminado hacia el logro de objetivos externos del individuo, el cual centra su atención principalmente en su propio bienestar. Otra orientación como resultado de condicionantes físicos relacionados con el rendimiento dependen fundamentalmente de componentes genéticos. Los componentes relacionados con salud no llegan a verse muy influenciados en esta orientación, pues aquí solo se logran asociar tan solo con un bajo riesgo de desarrollar prematuramente enfermedades derivadas de inactividad física. Sin embargo, hay niveles de actividad física manifestando declive en los parámetros necesarios para adquirir este tipo de condición, no se han llegado a cumplir, teniendo como referente lo mencionado por OMS, el Colegio Americano del Deporte, ACSM (por sus siglas en ingles), los cuales indican en sus documentos los minutos mínimos semana para realizar actividad física, estableciendo 150 minutos sumando actividades superiores a los 600 MET'S, además de su estrecha relación con el hipocampo.

Ante esta situación, es necesario aclarar cómo educar a nivel de pregrado en el caso de 
Universidades privadas en el marco seccional y se debe centrar sus bases en formación humanista enfocada a integralidad, complementando el desarrollo en todas sus dimensiones, resaltando entre ellas su calidad de vida (Nieto-López, García-Cantó \& Rosa-Guillamón, 2020). Es aquí donde se origina esta investigación, a nivel local, en el contexto bumangués al no conocer de forma precisa los efectos generadores de los niveles o tipos de actividad física. Por ello, el objeto de este trabajo es describir el efecto de actividad física como el comportamiento hipocampal asociado al rendimiento académico en estudiantes universitarios de facultades deportivas (Mujica-Sequera, 2020).

$\mathrm{Si}$ bien existen diversos ambientes académicos para estudiantes pertenecientes a planteles educativos, los resultados de este análisis evidenciaron un efecto directamente proporcional entre el protocolo de actividad física establecido con variables fisiológicas controladas y el comportamiento hipocampal (demanda de oxígeno progresiva ante ejercicio físico) a través de un análisis de cómputo donde se contrastaron datos estadísticamente significativos asociados a un desarrollo en la estructura cerebral que permitió un estímulorespuesta eficiente y coherente a estudiantes con un mayor promedio académico como indicador de aprendizaje.

Lo anteriormente mencionado es nuevo punto de partida para analizar a fondo herramientas en pro al mejoramiento del rendimiento académico, como en el desempeño del aprendizaje dependiente del hipocampo, para así garantizar el desarrollo integral del futuro profesional (Kirschner, Sweller, Kirschner \& Zambrano, 2018).

\section{Metodología}

Dada la situación por emergencia sanitaria ante el COVID-19, se disminuyó la muestra de esta investigación y se modificaron los parámetros de intervención. Por tal motivo, para este estudio cuasi experimental se realizó un muestreo no probabilístico por conveniencia (Ugalde Ramírez, 2019). La población elegida consistió en cincuenta y siete estudiantes del programa académico de pregrado en cultura física, deporte y recreación de la universidad
Santo Tomas, seccional Bucaramanga, en el marco del primer periodo académico del 2021, que cumplieron con los criterios de elegibilidad.

Acorde al protocolo de intervención inicialmente los estudiantes diligenciaron el consentimiento informado, aceptando su participación voluntaria en el estudio de manera digital a través de la plataforma de Microsoft forms (Sandoval, 2020). Seguido de esto, completaron el cuestionario mundial de actividad física, avalado por la OMS, el cual permitió medir la actividad física en cada uno de los dominios. El instrumento ha sido validado en varios países considerado idóneo para monitorear actividad física. A su vez, resultó ajustable para el contexto colombiano. Este instrumento evaluó la frecuencia semanal, intensidad, duración de actividad física realizada en tres dominios diferentes: ocupacional, transporte o desplazamiento y tiempo libre.

Otras variables por analizar fueron género, nivel socioeconómico. Descrito el proceso anterior, se asignaron los participantes pseudoaleatoriamente a cualquiera de los grupos de ejercicio aeróbico o control. Posteriormente, fueron emparejados por edad, genero, nivel de actividad física (auto informada resistencia media por semana). Con el fin de evitar diferencias en el estado físico o habilidades cognitivas entre grupos, al inicio del estudio, no hubo abandono durante la intervención.

En la segunda etapa de intervención se midió la memoria hipocampal y estriatal. Goldfarb (2016) como autor estableció la utilización de la tarea de búsqueda múltiple como facilitador del rendimiento visual. Dicha tarea de referencia contextual consistió en rápidas identificaciones de un objetivo incrustado en una matriz espacial, con una serie de distractores articulados. El objetivo es un estímulo "T", encontrándose este girado a $90^{\circ}$, ya sea a la derecha como a la izquierda, partiendo en congruencia dentro de la referencia espacial.

Por otro lado, los distractores se representaron por la forma "L", ubicados aleatoriamente en cuatro orientaciones diferentes, en correlación a una rotación angular de $0,90,180$ y 270 grados respectivamente. Además, los estímulos visuales, se presentaron sobre un fondo gris en un monitor estándar por estudiante, El objetivo apareció en un rango o 
área similar delimitada, dentro de una configuración sin variabilidad en los distractores por bloques. Cada pantalla constó de un solo objetivo o " $\mathrm{T}$ ", los elementos restantes, fueron los distractores y su aleatoriedad dependió de la medida estándar de la matriz. En el caso de los participantes, durante ejecución de dicha tarea, estuvieron regidos por ciertos parámetros o condiciones base. Principalmente, conservaron una proximidad o distancia al monitor de 50 centímetros. Así mismo, la fijación de la forma en cruz se presentó solo durante 0.5 segundos, seguida de otra presentación en pantalla.

En la tercera etapa de la medición guiada al participante, se dio una rápida respuesta con duraciones variables en intervalos, precediendo los rangos de la próxima prueba, en esta ocasión de tipo físico a través de cintas de correr, prueba submaximal del método Karvonen. Los participantes presionaron dos teclas en función de la frecuencia cardiaca máxima, ya sea derecha o izquierda, descrita anteriormente. Los elementos a nivel espacial se encontraron en una cuadricula la cual arrojaba en tiempo real a medida que el participante corría en la cinta, cuya distribución mostró cuatro elementos por cuadrante. Más aún, con el fin de completar una secuencia de prueba por bloques, formados estos últimos, por ensayos de pantalla repetida.

Finalmente, se consideró cualquier posible sesgo dentro de los factores, al aplicar tarea de búsqueda, entre ellos, la frecuencia de aparición por cuadrante, la distancia al punto de fijación, la dirección por patrón motor en respuestas similares contribuyó en la mejora a la reducción de la variabilidad en ocho configuraciones, únicas por cuadrante. Con respecto al lugar de aplicación, el área audiovisual del laboratorio del campus de la facultad de cultura física, deportes y recreación de la universidad Santo Tomas, seccional Bucaramanga. Este fue un espacio propicio respecto a reproducibilidad, fiabilidad misma de la intervención, en esta investigación.

\section{Resultados}

Se realizó un análisis descriptivo de las características de interés en población de estudio, las variables categóricas fueron descritas tanto con frecuencias como porcentajes. Las variables continuas se expresaron como media, desviación estándar cuando presentaron una distribución normal. En caso contrario, fueron descritas como mediana, rango intercuartílico. Se utilizaron los diferentes toolboxes del software Matlab para determinar diferencias estadísticamente significativas de las variables categóricas. Dentro del proceso de investigación se proyectó tomar datos, análisis, resultados en los meses de junio a diciembre, a raíz de los planes de contingencia por emergencia sanitaria del COVID-19, todas las fechas se adaptaron, se esperó aprobación paulatina del regreso presencial bajo el modelo de alternancia para intervención mediante el protocolo diseñado, análisis, resultados posteriores a fechas establecidas.

La muestra analizada mediante el protocolo inducido tanto de ejercicio físico asociado a VO2max, como las pruebas estándar de búsqueda " $T$ " dentro de actividad hipocampal evaluada, estuvo conformada por cincuenta y siete estudiantes de primer, segundo semestre, en el primer periodo del año 2021, pertenecientes a las cátedras deportivas de Universidad Santo Tomas, seccional Bucaramanga (22 hombres (38,06\%) 35 mujeres $(61,40 \%)$, ver Figura 1.

Acorde a la hipótesis planteada los resultados de este análisis evidenciaron un efecto directamente proporcional entre el protocolo de actividad física establecido con variables fisiológicas controladas y el comportamiento hipocampal (demanda de oxígeno progresiva ante ejercicio físico) a través de un análisis de cómputo donde se contrastaron datos estadísticamente significativos asociados a un desarrollo en la estructura cerebral que permitió un estímulo-respuesta eficiente y coherente mayor ante aquellos estudiantes que solo participaron de la prueba hipocampal.

El $42,11 \%$ de la población estudiada era físicamente inactiva, teniendo como parámetro el cumplimiento de las recomendaciones de actividad física expuesta por la organización mundial de salud. En cuanto a conducta sedentaria, el $73.68 \%$ de población dura más de 8 horas en estado de reposo, mientras el $26,32 \%$ dura menos de 8 horas sin este tipo de actividad. Cifras que permitieron contextualizar los bajos niveles de condición física y por ende bajos niveles de oxígeno en relación con el desempeño de estructuras cerebrales. Sin embargo se pudo esclarecer que aquellos con mejor 
desenvolvimiento hipocampal por actividad física tienden a obtener un mayor promedio académico como indicador de aprendizaje, siendo este último potenciado en mayor medida por la actividad física.

\section{Figura 1}

Descripción de las características sociodemográficas, de actividad física de los estudiantes de la Universidad Santo Tomas, seccional Bucaramanga $(n=57)$.

\begin{tabular}{lll}
\hline Características & n & \% \\
\hline Genero & & \\
\hline Masculino & 22 & 38.60 \\
\hline Femenino & 35 & 61.40 \\
\hline Nivel socioeconómico & & \\
\hline Bajo & 9 & 15.79 \\
\hline Medio & 44 & 77.19 \\
\hline Alto & 4 & 7.01 \\
\hline
\end{tabular}

Cumplimiento de las recomendaciones de la OMS sobre actividades para la salud $(n=91)$

\begin{tabular}{lll}
\hline Activo & 33 & 57.89 \\
\hline Inactivo & 24 & 42.11 \\
\hline Conducta sedentaria & & \\
\hline$>\mathbf{8}$ horas & 42 & 73.68 \\
\hline$<\mathbf{8}$ hora & 15 & 26.32 \\
\hline
\end{tabular}

Realización de actividad física en el tiempo libre

\begin{tabular}{lll}
\hline Si & 42 & 73.68 \\
\hline No & 15 & 26.32 \\
\hline
\end{tabular}

Realización de actividad física en el transporte

\begin{tabular}{|c|c|c|}
\hline Si & 52 & 91.23 \\
\hline No & 5 & 8.77 \\
\hline \multicolumn{3}{|c|}{ Realización de actividad física ocupacional } \\
\hline $\mathbf{S i}$ & 57 & 100 \\
\hline \multirow{2}{*}{ No } & 0 & 0 \\
\hline & Mediana & $\mathbf{R I} * *$ \\
\hline Edad & $18+$ & $2 * *$ \\
\hline Promedio academino & $4+$ & $2 * *$ \\
\hline $\begin{array}{l}\text { Total de minutos } \\
\text { actividad } \\
\text { fisica } 7 \text { semana }\end{array}$ & $120+$ & $105 * *$ \\
\hline $\begin{array}{ll}\text { Total } & \text { de } \\
\text { Met's7semana } & \end{array}$ & $520+$ & $660 * *$ \\
\hline
\end{tabular}

Nota. Análisis descriptivo de las características de interés en población de estudio, elaboración propia (2020).

\section{Conclusiones}

Los resultados de este análisis permitieron extraer una serie de conclusiones que deben ser contrastadas, medibles y puestas a prueba bajo el método científico en futuras investigaciones que relacionen el hipocampo, el aprendizaje y la actividad física específicamente. Ante la adversidad presentada por la pandemia la digitalización de formularios y el aplazamiento de la intervención, permitió restructurar la esencia metodológica y garantizó la eficiencia y rapidez a lo planteado inicialmente.
La adaptación del protocolo de actividad física se abordó como estado relacionado a mejorar las diferentes habilidades para acciones de tipo físico, muscular, motriz, tarea o actividad en el desempeño de forma regular de las memorias dependientes del hipocampo caracterizado por el dinamismo, energía o vitalidad de las personas, disfrutar del tiempo de ocio activo, afrontar las posibles emergencias imprevistas, evitando de esta forma ceder ante una plausible conducta sedentaria.

Se llegó a considerar como una aptitud o forma física evaluable dentro de un entorno físico, social, psicológico dentro de un amplio espectro entre una relación establecida con alta demanda en los niveles de actividad física, comportamiento hipocampal, garante de un proceso a establecer o caracterizar los indicadores asociados al impacto sobre el rendimiento académico en estudiantes universitarios (Deliens, Deforche, De Bourdeaudhuij \& Clarys, 2015).

Con base en postulados científicos, es pertinente utilizar softwares especializados como MATLAB pero con toolboxes más avanzados a los de esta investigación, con el fin de generar conceptos plausibles, acertados para crear diversas estrategias, con fines de contribuyan a elevar la calidad en futuras investigaciones. Es decir, se da relevancia a la necesidad de realizar estudios similares, con hipótesis más elaboradas a proyecciones de investigación en el área de la neurociencia.

\section{Referencias}

Anilema, J. A. S., Valdiviezo, D. G. P., Anilema, J. M. G., \& Paullán, M. J. C. (2020). Análisis estadístico del IMC en universitarios varones de la Escuela Superior Politécnica de Chimborazo. Lecturas: Educación Física y Deportes, 25(267).

Astudillo-García, C. I., \& Rojas-Russell, M. E. (2006). Autoeficacia y disposición al cambio para la realización de actividad física en estudiantes universitarios. Acta colombiana de Psicología, 9(1), 41-50.

Caballero, L. G. R., Delgado, J. C. S., Arenas, L. H. G., Díaz, J. S. M., \& López, S. M. S. (2020). Physical activity, body composition and musculoskeletal capacity of teenage students from Floridablanca, Colombia. Revista Cubana de Investigaciones Biomédicas, 39(1), 1-17. 
Deliens, T., Deforche, B., De Bourdeaudhuij, I., \& Clarys, P. (2015). Determinants of physical activity and sedentary behaviour in university students: a qualitative study using focus group discussions. BMC public health, 15(1), $1-9$.

Duque-Fernández, L. M., Ornelas-Contreras, M., \& BenavidesPando, E. V. (2020). Actividad física y su relación con el envejecimiento y la capacidad funcional: una revisión de la literatura de investigación. Psicología y Salud, 30(1), 45-57.

Goldfarb, E. V., Chun, M. M., \& Phelps, E. A. (2016). Memoryguided attention: independent contributions of the hippocampus and striatum. Neuron, 89(2), 317-324.

Guadalupe, J. E., Amaya, J. R., \& Condo, G. Á. (2015). Factores de riesgo y prevención del estrés académico en estudiantes universitarios de la UNEMI, Milagro. YACHANA Revista Científica, 4(2).

Jiménez, D. F. L. (2007). La naturaleza de las tecnologías de información y comunicación: las TIC como determinantes de la organización y de la sociedad de la información. Palabra Clave, 10(1).

Jung, M. E., Bray, S. R., \& Ginis, K. A. M. (2008). Behavior changes and the freshman 15: tracking physical activity and dietary patterns in 1st-year university women. Journal of American College Health, 56(5), 523530 .

Kirschner, P. A., Sweller, J., Kirschner, F., \& Zambrano, J. (2018). From cognitive load theory to collaborative cognitive load theory. International Journal of Computer-Supported Collaborative Learning, 13(2), 213-233.

Mujica-Sequera, R. M. (2020). La Enseñanza Tecnoemocional en la Educación del Siglo XXI. Revista TecnológicaEducativa Docentes 2.0, 9(2), 71-78.

Nieto-López, L., García-Cantó, E., \& Rosa-Guillamón, A. (2020). Relación entre nivel de condición física y percepción de la calidad de vida relacionada con la salud en adolescentes del sureste español. Revista de la Facultad de Medicina, 68(4).

Polo-Zegarra, K. E. (2020). Estilo de vida y factores biosocioculturales en universitarios de la escuela profesional

Rojas, G., Salas, R., \& Jimenez, C. (2006). Estilos de aprendizaje $\mathrm{y}$ estilos de pensamiento entre estudiantes universitarios. Estudios pedagógicos (Valdivia), 32(1), $49-75$.

Sandoval, C. H. (2020). La educación en tiempo del Covid-19 herramientas TIC: El nuevo rol Docente en el fortalecimiento del proceso enseñanza aprendizaje de las prácticas educativa innovadoras. Revista TecnológicaEducativa Docentes 2.0, 9(2), 24-31.

Ugalde-Ramírez, J. A. (2019). Revisión sistemática y metaanálisis de los efectos del ejercicio físico sobre el volumen del hipocampo y materia gris del cerebro humano.
Wing-Kwan, M. Y., Bray, S. R. \& Martin Ginis, K. A. (2009). Predicting physical activity of first-year university students: An application of the theory of planned behavior. Journal of American College Health, 58(1), 4555. 